\title{
TDoA-based outdoor positioning in a public LoRa network
}

\author{
Nico Podevijn ${ }^{1}$, Jens Trogh ${ }^{1}$, Abdulkadir Karaagac ${ }^{2}$, Jetmir Haxhibeqiri ${ }^{2}$, \\ Jeroen Hoebeke ${ }^{2}$, Luc Martens ${ }^{1}$, Pieter Suanet ${ }^{3}$, Kim Hendrikse, David Plets ${ }^{1}$, Wout Joseph ${ }^{1}$ \\ ${ }^{1}$ Department of Information Technology, University of Ghent, IMEC, WAVES, Ghent, Belgium, nico.podevijn@ugent.be \\ ${ }^{2}$ Department of Information Technology, University of Ghent, IMEC, IDLab, Ghent, Belgium \\ ${ }^{3}$ Aucxis crba, Stekene, Belgium
}

\begin{abstract}
The performance of LoRa Geo-location for outdoor tracking purposes has been evaluated on a public LoRaWAN network. Time Difference of Arrival (TDOA) localization accuracy, probability and update frequency were evaluated for different trajectories (walking, cycling, driving) and LoRa spreading factors. A median accuracy of $200 \mathrm{~m}$ was obtained and in $90 \%$ of the cases the error was less then $480 \mathrm{~m}$.
\end{abstract}

Index Terms-LoRA, Geo-Location, Tracking.

\section{INTRODUCTION}

Currently most track and trace solutions rely on GPS. Although providing accurate real time location updates, it has a disadvantage of consuming a fair amount of power and therefore a regular battery replacement is needed. As of today, public LoRaWAN networks are being deployed with Geo-location functionality which are capable of locating nodes using the Time difference of arrival (TDOA) technique [1]. The Geo-localization accuracy is worse than GPS but a main advantage is the fact nodes are able to operate for years without replacing the battery. Other advantages are the fact that a single technology is used for both communication and localization, making it a low-cost alternative. An example application for this alternative is tracking of goods between industrial warehouses. The novelties of this paper are:

- First-time quantification of the TDOA Geo-location performance on a public deployed LoRa network for different scenarios (walking, cycling, driving).

- Determination of the best spreading factor to use

This paper is organized as follows. Section II describes related work in this new research area. In section III, the measurement setup, evaluated scenarios and estimation of TDOA performance are discussed. Results of TDOA performance and selection of best spreading factor to use are discussed in section IV. We summarize our observations and work in Section V.

\section{RELATED WORK}

In [4] a private LoRaWAN network with 4 gateways (each $2-3 \mathrm{~km}$ apart) was deployed with TDOA capabilities and the geolocalisation was performed for stationary nodes. The reported mean accuracy was around $100 \mathrm{~m}$. The difference with our work lies in the fact that we investigate and improve the performance on a publicly deployed network with mobile

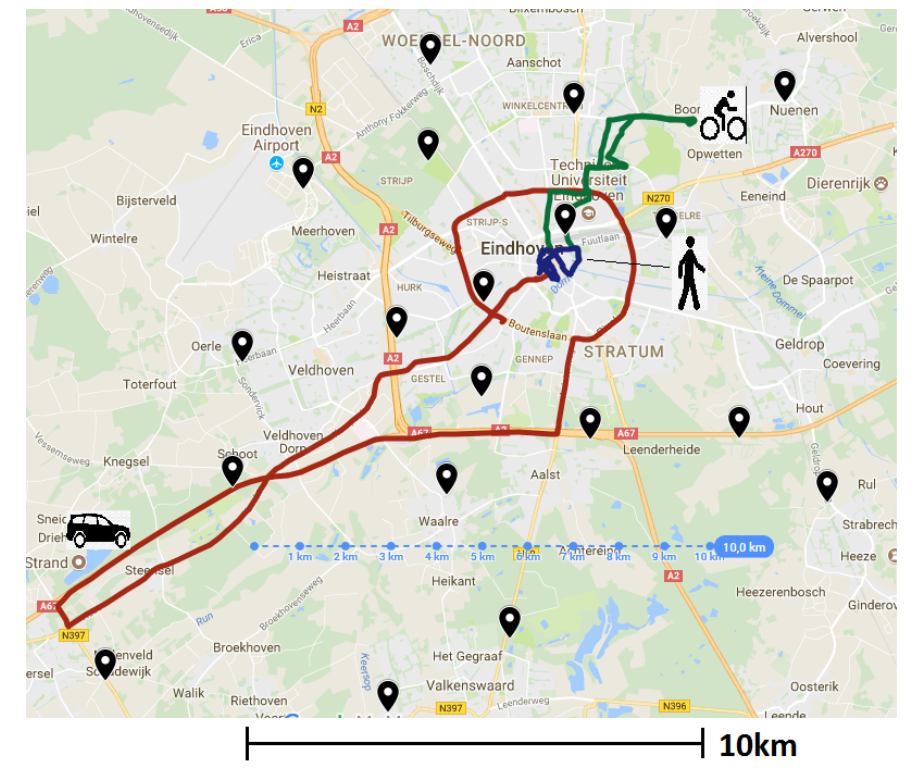

Fig. 1. Walking (blue), cycling (green) and driving (red) Trajectories. The black pointers are the gateway locations

nodes. To our knowledge, this has never been performed, mainly due to the fact LoRaWAN public networks with TDOA capabilities have only become recently public available [1].

\section{MATERIALS AND Methods}

\section{A. Configuration}

Six LoRa nodes were configured and provisioned in the public LoRa network in the Netherlands. For each node ADR (Adaptive Data Rate) wad disabled to force a fixed spreading factor (SF7-SF12). Uplinked data size was set to 2 bytes (e.g. for sensor data and battery level status). Our implemented application server recorded the JSON messages from the network server which hold the location estimates (in latitude and longitude degrees) for each device.

Since the time on air depends on the spreading factor used [5], the period between uplinks was set accordingly in order to respect the duty cycle of $1 \%$ in the $868 \mathrm{MHz}$ industrial, scientific and medical (ISM) band. Table I shows which transmission interval or period between transmissions was used for each node. 
TABLE I

IMPLEMENTED TRANSMISSION PERIOD FOR EACH SF

\begin{tabular}{cccc}
\hline \hline SF & Time on Air [ms] & Duty cycle & Period [s] \\
\hline 7 & 46 & 0.01 & 4.6 \\
8 & 82 & 0.01 & 8.2 \\
9 & 165 & 0.01 & 16.5 \\
10 & 289 & 0.01 & 28.9 \\
11 & 660 & 0.01 & 66 \\
12 & 1155 & 0.01 & 116 \\
\hline
\end{tabular}

However, not every transmission will lead to a location update. Only when a data transmission is received by 3 or more gateways, the network is able to estimate the location of the node using the TDOA (Time Difference of Arrival) technique [1]. The estimated locations are calculated by the LoRa network server and are therefore not estimated by us. The location estimate is given in latitude and longitude degrees.

\section{B. Scenarios}

Measurements were performed in and around Eindhoven, an urban city in the Netherlands. The nodes were carried along 3 different routes: Walking, Cycling and Driving: See figure 1. The ground truth (with time stamp) of each trajectory was recorded using a GPS logger. The characteristic of the three trajectories such as average speed, maximum speed, travelled distance and duration are summarized in Table II.

TABLE II

MEASURED TRAJECTORIES AND THEIR CHARACTERISATION

\begin{tabular}{ccccc}
\hline \hline Tajectory & Duration & Distance & Max.Speed Speed & Avg. Speed \\
\hline Walking & $60 \mathrm{~min}$ & $4.4 \mathrm{~km}$ & $6 \mathrm{~km} / \mathrm{h}$ & $4.4 \mathrm{~km} / \mathrm{h}$ \\
Cycling & $60 \mathrm{~min}$ & $12.1 \mathrm{~km}$ & $21 \mathrm{~km} / \mathrm{h}$ & $12.1 \mathrm{~km} / \mathrm{h}$ \\
Driving & $60 \mathrm{~min}$ & $38.7 \mathrm{~km}$ & $137 \mathrm{~km} / \mathrm{h}$ & $38.7 \mathrm{~km} / \mathrm{h}$ \\
\hline
\end{tabular}

\section{Determining location update rate}

We evaluated the update rate of the geo-location updates as follows. The amount of transmissions during the measurement period was measured. From these transmissions, we record the number of packets received by 1 or more (up to 3) gateways. Finally, we record the number of geo-updates in the measured time span. Dividing the time duration by the number of geolocation updates reveals the average location update period. We then select the best spreading factor $S F_{\text {opt }}$ as the one which gives the smallest update period:

$$
S F_{\text {opt }}=\underset{S F x}{\operatorname{argmin}} \frac{T D}{N R_{S F x}}, \text { with } x=7-12
$$

With TD the measurement time and $N R_{S F x}$ the number of geo-updates collected during that time on the considered spreading factor.

\section{Determining location accuracy}

The received TDOA location estimates were compared with the ground truth location for each trajectory and node. This

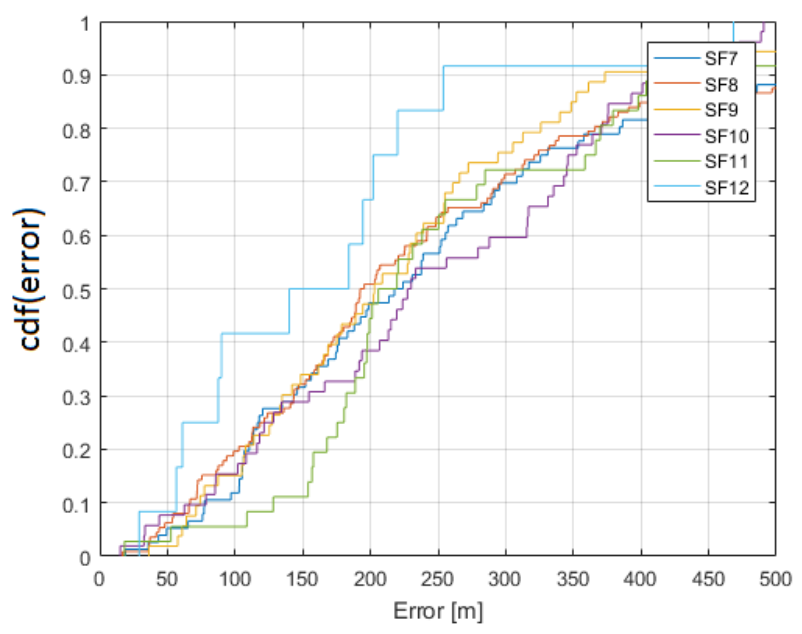

Fig. 2. CDF of localisation error for walking route

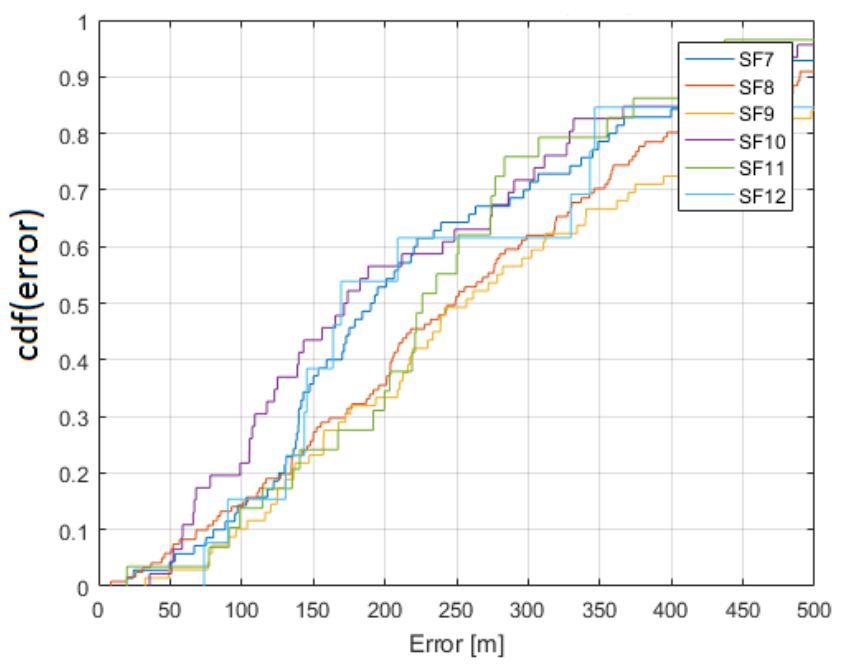

Fig. 3. CDF of localisation error for cycling route

was done with the help of the obtained time stamps from both the received estimates and the GPS logger. The positioning errors were calculated for each combination of node (SF) and each trajectory. From these errors we were able to compute the cumulative probability distribition function (CDF), standard deviation, median error and 90th percentile error.

\section{RESUlts}

\section{A. Comparison of TDOA location estimates for different SFs} and different ways of transportation

In Table IV the number of location updates for each trajectory and SF is presented. For SF7 the uplink period is minimal (Table I). Hence we expect this SF providing most updates/hour. However, one must take into account that for low SFs the effective communication range is relatively small. Therefore, the probability of reception at 3 gateways is smaller when compared to using a high SF. The highest number of 
TABLE III

ERRORS FOR DIFFERENT SPREADING FACTOR AND TRAJECTORIES

\begin{tabular}{|c|c|c|c|c|c|c|c|c|c|c|c|c|}
\hline & \multicolumn{4}{|c|}{ Median error $[\mathrm{m}]$} & \multicolumn{4}{|c|}{ 90th percentile error $[\mathrm{m}]$} & \multicolumn{4}{|c|}{ Std.Deviation [m] } \\
\hline & Walking & Cycling & Driving & All Traj. & Walking & Cycling & Driving & All Traj. & Walking & Cycling & Driving & All Traj. \\
\hline SF7 & 221 & 191 & 108 & 193 & 504 & 460 & 328 & 470 & 164 & 168 & 112 & 163 \\
\hline SF8 & 194 & 249 & 116 & 207 & 579 & 490 & 324 & 503 & 216 & 323 & 119 & 270 \\
\hline SF9 & 203 & 257 & 192 & 230 & 383 & 552 & 410 & 515 & 127 & 198 & -130 & 171 \\
\hline SF10 & 229 & 173 & 233 & 215 & 437 & 425 & 482 & 430 & 134 & 142 & 146 & 138 \\
\hline SF11 & 213 & 226 & 239 & 226 & 413 & 419 & 623 & 435 & 172 & 442 & 201 & 303 \\
\hline SF12 & 162 & 169 & 109 & 164 & 318 & 538 & 195 & 420 & 120 & 168 & 50 & 144 \\
\hline All & 207 & 221 & 145 & 211 & 470 & 489 & 400 & 484 & 175 & 264 & 145 & 219 \\
\hline
\end{tabular}

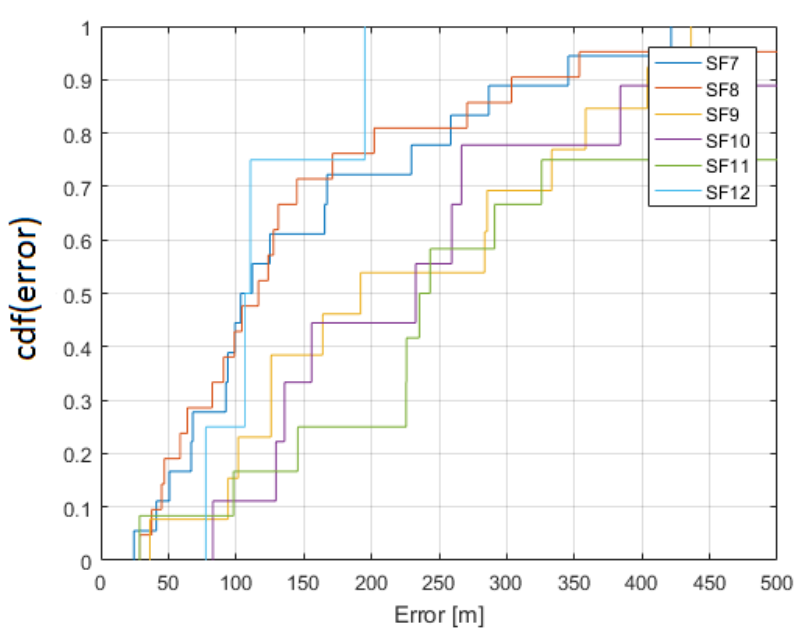

Fig. 4. CDF of localisation error for driving route

location updates was found when using SF8 for all trajectories $(=254)$. The number of location updates is comparable for the walking and cycling trajectories (112 vs. 121 for SF8). For the car route we note a much lower number of location updates (21 for SF8). This is due to the fact of the metal frame of the vehicle attenuating the RF transmissions in the $868 \mathrm{MHz}$ ISM band [2] and the increased mobility vs. walking and cycling trajectories.

TABLE IV

NUMBER OF LOCATION UPDATES IN ONE HOUR

\begin{tabular}{cccc}
\hline \hline SF/Route & Walking & Cycling & Driving \\
\hline 7 & 76 & 70 & 18 \\
8 & 112 & 121 & 21 \\
9 & 53 & 69 & 13 \\
10 & 52 & 46 & 9 \\
11 & 36 & 29 & 12 \\
12 & 12 & 13 & 4 \\
\hline
\end{tabular}

Figure 5 shows the number of geo location updates normalized to the known number of transmissions for the walking route. $\mathrm{p}(\mathrm{RX} 1 \mathrm{GW})$ is the probability of reception at 1 or more gateways, $\mathrm{p}(\mathrm{RX} 3 \mathrm{GW})$ is the probability of reception at 3 or

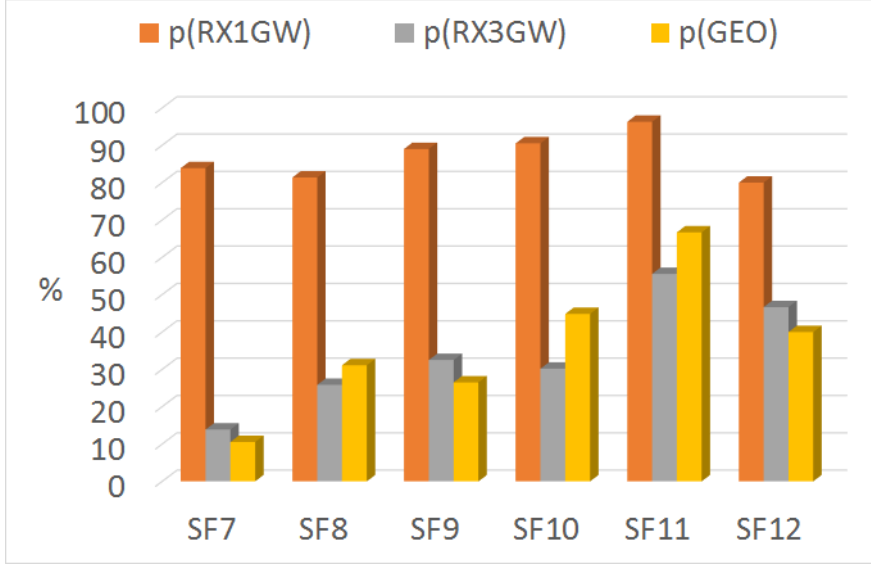

Fig. 5. Probabilities of a location update for different spreading factors: $\mathrm{p}(\mathrm{RX} 1 \mathrm{GW})=$ probability of reception at 1 or more Gateway, $\mathrm{p}(\mathrm{RX} 3 \mathrm{GW})=$ probability of reception at 3 or more gateways, $\mathrm{p}(\mathrm{GEO})=$ probability of a location update.

more gateways, $\mathrm{p}(\mathrm{GEO})$ is the probability of a geo-location update.

With the exception of SF12, we observe that reception and location update probabilities increase with higher SF. This is due to the fact higher spreading factors have higher RX sensitivity and thus increase the communication range. Therefore transmissions are more likely to be received by the LoRa Gateways. The highest probability of a location update is obtained when using SF11. Similar trends were found for the cycling and driving routes. Further investigation is needed why this trend does not persist for SF12.

The TDOA error distribution for the walking, cycling and driving route is shown in Figures 2, 3, 4 respectively. The median, 90th percentile and standard deviation of the error can be found in Table III. From this table, we can observe that the overal median error is around $200 \mathrm{~m}$ and in $90 \%$ of the cases this error is less then $500 \mathrm{~m}$. Altough there is no trend in estimation error depending on the SF and/or trajectory, the median error was minimal for all trajectories when using SF12 $(164 \mathrm{~m})$.

In Figure 6 the tracking results are shown for the walking route when using SF8. In our future work we will implement 


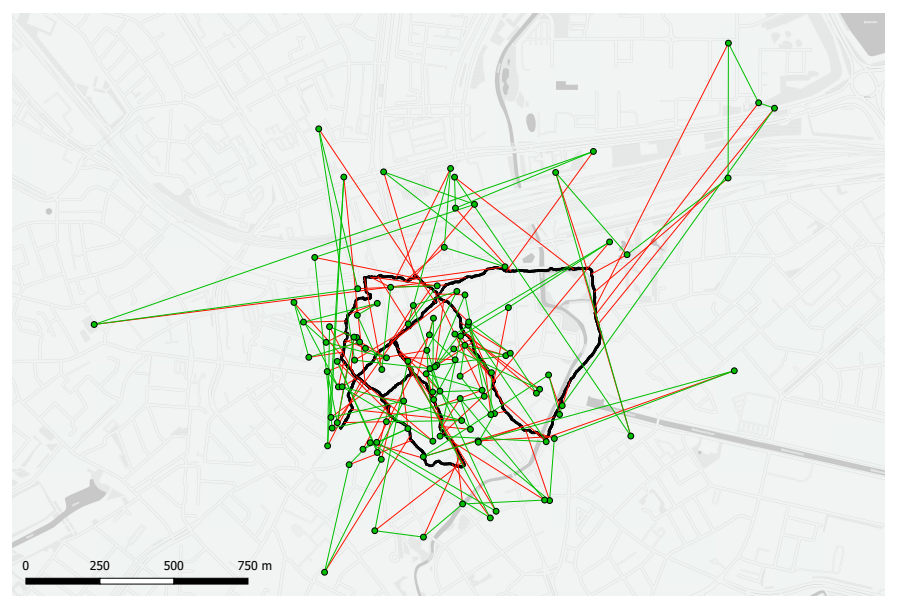

Fig. 6. Tracking results for walking route with SF8. Black line= ground truth trajectory; Green dots= estimated TDOA locations; Red Lines = error between estimation and ground truth

an algorithm [3] which takes into account the street map and limited mobility of the node to discard the outliers and improve the localization.

\section{CONCLUSION}

In this paper we investigated the tracking performance of nodes using a public LoRaWAN network with TDOA capabilities. The median error was around $200 \mathrm{~m}$ and in $90 \%$ of the cases the error was less then $500 \mathrm{~m}$ over all SFs and trajectories. No trend was found on the performance of localization error when varying the mobility or the spreading factor, but in general the best localization accuracies were obtained for SF12. We obtained the highest probability of a location update when using spreading factor 11 and obtained the most location updates when using spreading factor 8. As part of our future work we will implement an algorithm which takes into account the street map and known mobility to further enhance localization accuracy.

\section{ACKNOWLEDGEMENT}

This research is part of the HYCOWARE project realized in collaboration with IMEC. Project partners are Egemin, Aucxis and Intation, with project support from VLAIO (Flanders Innovation and Entrepreneurship).

\section{REFERENCES}

[1] KPN, LoRa Geolocation-Getting started, Version 1.3, Netherlands, 2017.

[2] E. Tanghe, W. Joseph, L. Verloock, and L. Martens, Evaluation of Vehicle Penetration Loss at Wireless Communication Frequencies, IEEE Trans. Veh. Techn., vol 57, no. 3, pp. 2036 2041, July 2008.

[3] J. Trogh, D. Plets, L. Martens and W. Joseph, "Advanced real-time indoor tracking based on the viterbi algorithm and semantic data," 2015 International Journal of Distributed Sensor Networks, London, 2015.

[4] B. C. Fargas and M. N. Petersen, "GPS-free geolocation using LoRa in low-power WANs," 2017 Global Internet of Things Summit (GIoTS), Geneva, 2017, pp. 1-6.

[5] LoRa Modem Designers Guide, https://www.semtech.com/images/ datasheet/LoraDesignGuide_STD.pdf. 